\title{
Energy Efficient Smart Sensor Network Routing Protocol using Node Scheduling
}

\author{
Sadiya Jameel \\ M. Tech. Scholar \\ CSE Dept
}

\author{
Zuber Farooqui \\ Asst. Prof. \\ CSE Dept
}

\begin{abstract}
Human needs are going more technology dependent in last decade. Technology touches live everywhere and also reach the area where humans unable to reach. One of Such important application is Wireless Sensor Networks (WSNs). This helps to collect data from different conditions where human faces challenges to gather information about atmosphere or environment. Apart of its advantage, WSN suffer from many different challenges like limited sensor node electric capacity, pattern of node deployment, data sending mechanism etc. To manage WSN with optimum resource utilization various data transmission methods have been given in recent years. This paper proposed another better method for improving network life span and increase stability of WSN. Proposed method manages all sensor nodes energy power within intelligence manor by using ON-OFF scheduling of node data transmission. Few nodes in ON state sense data and send to base station for one round of data transmission while other nodes are in power saving OFF mode by doing nothing neither sense nor transmit data. But for next round nodes are in ON status nodes goes to OFF power saving modes to save power and OFF mode nodes sense and send data to base station. This method is compared with other technique using same parameter while simulation and proposed method outperform better in all aspects of WSN comparison.
\end{abstract}

\section{Keywords}

Energy efficient, Data transmission protocol, ON mode, OFF mode, WSN, scheduling

\section{INTRODUCTION}

The rapid growth of technology is very helpful to make easy life. It opens new way and resources for analysis environment within critical situations. Wireless sensor network (WSN) prove a great change in this field. WSN network make simple to capture and collect useful data from such places where any human found difficulties to survive [1]. WSN has large application area including smart office with automatic power switches, traffic monitoring system with intelligent signaling, military surveillance without human need, health monitoring system to collect periodical data etc.

Such Sensor networks primarily consist of very small, low power sensor hardware circuit as shown in figure 1. These nodes are placed randomly within desire location to collect special data from sensor about atmosphere. These small nodes are able to gather data, process if required and transmit this data to base station for further decision making. WSN have many research issues need to be solve for better network utilization [2]. Such types of network have to perform within dynamic manner where node may join or left network any time this reason make WSN difficult and performance of total packet transmission degrades. So it's very needful to design protocol able to transmit data with optimum utilize energy of nodes and enhance total life time of network for better performance. Some algorithms are very sensitive if serious node stop its function this result to overall network failure or may disconnect some nodes from base station communication [3]. Distributed protocols are more effective to handle such situations.

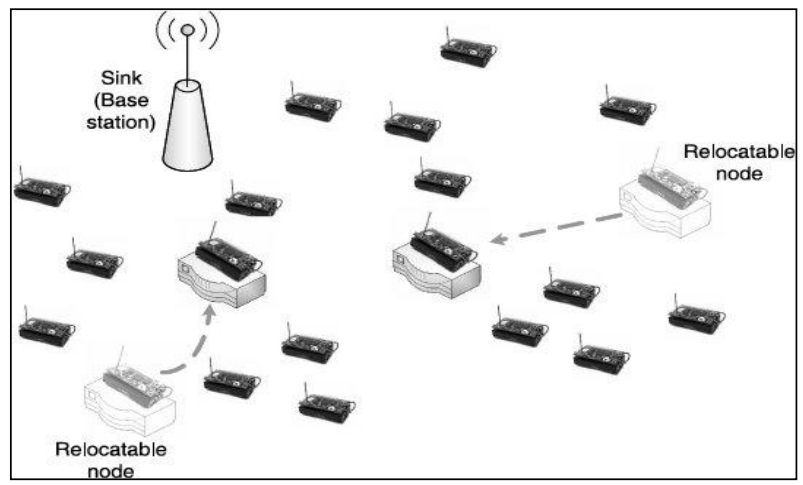

Figure 1 Sample WSN architecture

For optimum energy utilization clustering methods are send data of some nodes by aggregation method. A cluster head combines data of all members within a single packet and sends to base station hence member nodes are need to send information only to its near cluster head with less memory power and only cluster head send all data in aggregated manor so all members nodes save their energy for short distance communication. This method also help for scalability if more nodes are join network they can join existing cluster or may create a new cluster as clustering algorithm specified within network. This paper gives overall study of existing methods and reviews its advantages and drawback on basis of this analysis proposes new method for enhance performance of WSN.

Some clustering protocols such as LEACH, SEP, DEEC [4-8] are somehow get better performance in WSN conditions. By considering these methods and combining advantage of all these method within a single protocol could be very effective for overall enhancement.

\section{RELATED WORK}

LEACH [4] could be a ranked cluster algorithmic rule for considered usage of energy within the network. LEACH uses irregular rotation of the nearer cluster head. LEACH performs well in uniform conditions. In LEACH each node has same chance to become a cluster head. However, LEACH isn't compatible for heterogeneous conditions. SEP could be a two level heterogeneous protocol introducing two kinds of nodes, traditional nodes and advance nodes. Advance nodes have a lot of energy than traditional nodes. In SEP each nodes ([5] advance nodes) have weighted chance to become cluster 
head. Advance nodes have a lot of probabilities to become cluster head than traditional nodes. SEP doesn't guarantee economical installation of nodes.

WSN need to be scale as required in such conditions hierarchical clustering is better way to deal with data transmission. Hierarchical techniques have extra benefit as efficient communication with good energy utilization. Such type of applications nodes having extra energy than normal nodes used to send sensed information. There are various protocol have been given such as LEACH [4], TEEN [10], APTEEN [11], PEGASIS[9] these method used advance node for better energy consumption.

Data load transmitted over network is very essential parameter for any network. This parameter is check and optimized during work on paper [12] by authors as clustering with load balancing for WSN network. Author verify density of cluster according to distance from base station. Hence if nodes are near to base station than density of clusters are low and for nodes are long distance from base station are from cluster with more density to optimum energy use. This method improved total life time of network.

Another previous technique consider more than one parameters as WCA [13] single hop distance. Cluster head selection is base on different parameters like one hop neighbors, remaining battery power and speed to node movement. This method need to calculate weight factor during each round hence need more power for calculation. Hence extra effort has to apply for cluster formation this overhead control the efficient of WCA method.

Basic method used for clustering sensor nodes k-clustering [14] used most k-hop neighbors to form cluster.

The ESAC [15] method uses main advantages of above mention methods and make more effective clustering method. This method uses cluster size as requirement of WSN condition. This restricts to create large size cluster to efficient clustering up-to two hop node distances. If cluster head died than new cluster head selected from this cluster member only. So new cluster formation take place only within current members other nodes are not affected by this cluster head selection. But two-hop distance clustering is not good idea for cluster formation.

\section{PROPOSED WORK}

This section give proposes new method this works on both environment homogenous as well as for heterogeneous network very well. This work mainly focus for minimize total network power consumption with quality service to improve stability of network and total network life time. Stability of network means when first node died within any network it should be take long enough time to stop working for first node.

To accomplish mention objective here introduce the concept of node pairing. Senor nodes within any network are deploying random positions. Due this random node distribution some nodes are placed very near to each other with respective to their distance. These nodes sense same information and transmit to base station but base station not need for redundant information there is extra work load for same repeated data. Hence proposed work removes this repeated information by pairing nodes within minimum distance to each other compare to predefine threshold value. There are also some improvements in clustering technique for selection cluster head $(\mathrm{CH})$ with selection parameter remaining energy of nodes. More details are given as below.
Once nodes are paired to each other according to distance, only one of them is in working state (ON state) at a same time and other node is in not working state (OFF state) during that round. Hence only one node from two is utilized energy power while other saves its energy by non active mode.

Node Pairing

On setup of any WSN network base station manages pairing process by locating position of all sensor nodes by collecting hello message from all nodes. Using geographical location position a base station to get pair for nearest node if exists. The nearest node makes pair as directed by base station according their distance position. Such two nodes get connected as pair through their node IDs. Overall mechanism is given in algorithm 3.

Cluster Heads Election

The sensor network can be homogenous or heterogeneous so proposed method is planned by considering both type of networks and effectively solve this issue by selecting cluster head higher energy node with higher selection probability. Cluster node must have higher energy power as well as must have higher energy level than whole network average energy. The systematic algorithm can be given as algorithm 2 .

\subsection{Proposed Algorithms}

\section{Algorithm 1: SensingRegionSelection0}

This algorithm determines the region where clustering is required.

Input: Position of the sensor nodes and BS.

Output: Direct transmission region, Clustering region.

begin

1 for a given simulation time $\mathrm{T}$ do

2 BS broadcasts HELLO packets periodically.

3 if a sensor node listen HELLO packet then

4 Reply with residual energy.

5 if the RSSI of the received signal RSSIch

then

6 No need to form clusters.

7 else

8 Clustering occurs according to ClusterSelection 0

end

\section{Algorithm 2: ClusterSelection0}

This algorithm grouped the sensor nodes into clusters.

Input: No. of Sensor nodes, Initial node energy, probability (p), No of rounds.

Output: Cluster heads, Clusters.

begin

1 if any node is alive then

2 follow nodePaining 0 to pair with closest neighbour. 
3 follownodeClass StatusSetup (to decide about the on nodes.

4 if Node.Class $=\mathrm{ON}$ for any node then

5 for each round do

$6 \mathrm{BS}$ choose $\mathrm{CHs}$ following $\mathrm{CH}$ setup $\mathrm{O}$

7 if a node is Cluster Head then

8 Broadcasts its $\mathrm{CH}$ advertisement message

9 All non-CHs on nodes, sends joining request message to that $\mathrm{CH}$, from which it received the highest RSSI.

10 Cluster head accepts the joining request.

11 Forms respective clusters.

End

\section{Algorithm 3: NodePairing0}

This algorithm coupled the nodes.

Input: No. of Sensor nodes, RSS

Output: Set of paired-node

begin

1 A Base station gets closest nodes by their location and find Closest Neighbor nodes.

2 The neighbors answer consistently.

3 The neighbor with the strongest RSSI is selected as the next node.

4 Node Status $=$ PAIRED

5 Base station stored Node Paired ID

end

\section{Algorithm 4 NodeClassStatusSetup0}

This algorithm set the mode of node whether switch to off mode or on mode for this round.

Input: No. of Sensor nodes, Node residual energy.

Output: Off node, On node.

begin

1 Get $\mathrm{N}$ paired node sets in the network.

2 if node $=$ PAIRED then

3 for each pair set do

4 Node broadcasts an Energy Msg message to its neighboring node.

5 Receiving Energy Msg from its neighbouning node, updates its own Neighbor Table.

6 if $\mathrm{E}(\mathrm{r})$ node $>\mathrm{E}(\mathrm{r})$ neighbour

then

7 Set Node.Class $=$ ON and Node.Status $=1$

8 else

9 The node Broadcasts go To Off message to neighbours.

10 Node.Class = OFF

11 Set Node.Status $=0$

12 Nodes receive their status update
13 for a pair do

14 if neighbor node is dead then

15 Another node of that pair $\mathrm{On}$ for remaining rounds.

16 else Node.Class = ON for whole networklife time.

end

\section{Algorithm 5 CHsetup 0}

This algorithm selects the powerful nodes as cluster head

Input: No. of Sensor nodes, Initial node energy, Node Residual Energy, Energy Consumption rate, No of rounds.

Output: Cluster heads.

begin

$1 \mathrm{BS}$ receives the status of the Eresi from all nodes.

2 Then based on this BS computes Eavg of the on nodes.

3 Broadcasts the Eayg

4 for each node having Eresi. Eavg

do

5 Include in the set of eligible $\mathrm{CHs}$

6 if a node finds its Eresi. Eavg then

7 it sends a request message to find eligible neighbours.

8 The 1-hop neighbour nodes closer to that node send a reply with their

Eecr of $r-1$.

9 The node which has min Eecr and with Eresi Eavg is selected as $\mathrm{CH}$ node.

End

\section{SIMULATION AND RESULTS}

The proposed method is analysis under all standard parameters within WSN network and compared to previous protocol given to energy utilization improvement. This work is simulate using network area measurement $100 \mathrm{~m}$ by $100 \mathrm{~m}$ same for all compared clustering methods. The proposed method is observed in both homogeneous and heterogeneous architecture same for LEACH and SEP hence normal and advanced nodes are place randomly within network area. For check nodes location position horizontal and vertical direction axes are used. Each sensor element assigned random coordinate positions for location placement. There are 100 nodes are deployed in network area from these nodes ' $\mathrm{m}$ ' percentage nodes are advance nodes having ' $a$ ' percentage extra energy in compare to normal nodes. The base station is placed at center position of any one side of network field hence every sensor node is approximately $70 \mathrm{~m}$ distance long from base station. The simulation for this run for 5000 rounds in MATLAB R2010b.During simulation to get exact compare proposed work result with LEACH [16], SEP [17] and MGEAR [18] methods total advance and normal nodes with different remaining battery power kept same. The different statics used in model are given in Table 1. 
Table 1: Parameters used in implementation

\begin{tabular}{|c|c|}
\hline $\mathrm{p}$ & 0.1 \\
\hline $\mathrm{n}$ & 100 \\
\hline Eo & $0.5 \mathrm{~J}$ \\
\hline ETX & $50 \mathrm{~nJ} / \mathrm{bit}$ \\
\hline ERX & $50 \mathrm{~nJ} / \mathrm{bit}$ \\
\hline $\mathrm{fs}$ & $10 \mathrm{pJ} / \mathrm{bit} / \mathrm{m} 2$ \\
\hline $\mathrm{mp}$ & $0.0013 \mathrm{pJ} / \mathrm{bit} / \mathrm{m} 4$ \\
\hline $\mathrm{EDA}$ & $5 \mathrm{~nJ} / \mathrm{bit}$ \\
\hline $\mathrm{r}$ & 5000 \\
\hline
\end{tabular}

The results for LEACH, SEP, M-GEAR are compared with proposed method under two level heterogeneous network structures. The simulations measured using three special factors settings. $1 . \mathrm{m}=0.1$ and $\mathrm{a}=1$ means $10 \%$ nodes are advance nodes in network with 1 times more energy than normal nodes.

2. $m=0.3$ and $a=1$ means $30 \%$ nodes are advance nodes in network with 1 times more energy than normal nodes.

3. $m=0.3$ and $a=3$ means $30 \%$ nodes are advance nodes in network with 3 times more energy than normal nodes.

\subsection{Stability of network}

The result generated through simulation specified that proposed scheme extend the stability of network in compare to other methods. As given protocol keep some nodes in OFF mode and save energy of those nodes alternatively each round. Proposed method select $\mathrm{CH}$ is basis on energy consumption rate with considers overall network average energy. Due to this given method able to keep first node alive for very long time to improve stability period. Simulation outcomes are given within Figure 2, Figure 3 and Figure 4.

From Figure 2 clears that first node stop working during LEACH protocol in 976 rounds, in M-GEAR completion of 783 rounds, in SEP after 985 rounds but proposed work maintain all nodes working for 1470 rounds.

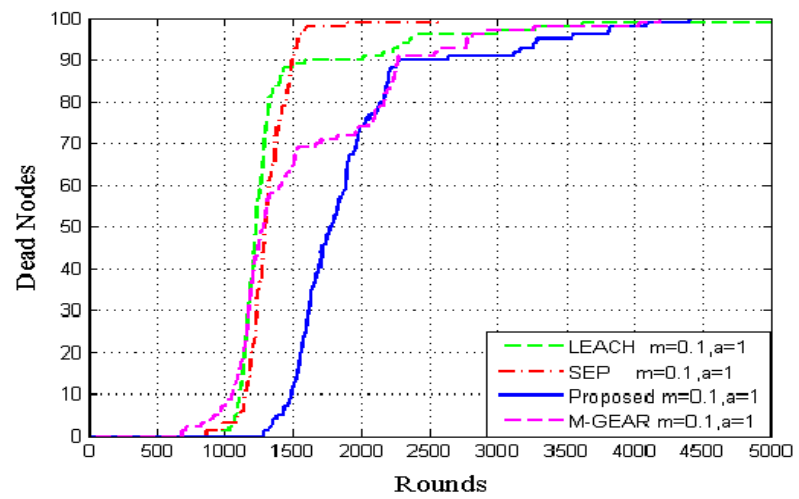

Fig 2. Comparison of proposed algorithm with existing in existence of heterogeneity $(m=0.1, a=1)$

Hence proposed method achieves 1.32 and 1.35 times enhanced result with compare to LEACH also 1.57 and 1.58 times enhanced result with compare to M-GEAR and 1.16 and 1.02 times enhanced result with compare to SEP. Study of the graphs is obvious that given method not just achieve good result than other, however distribution of power expenditure as well consistent.

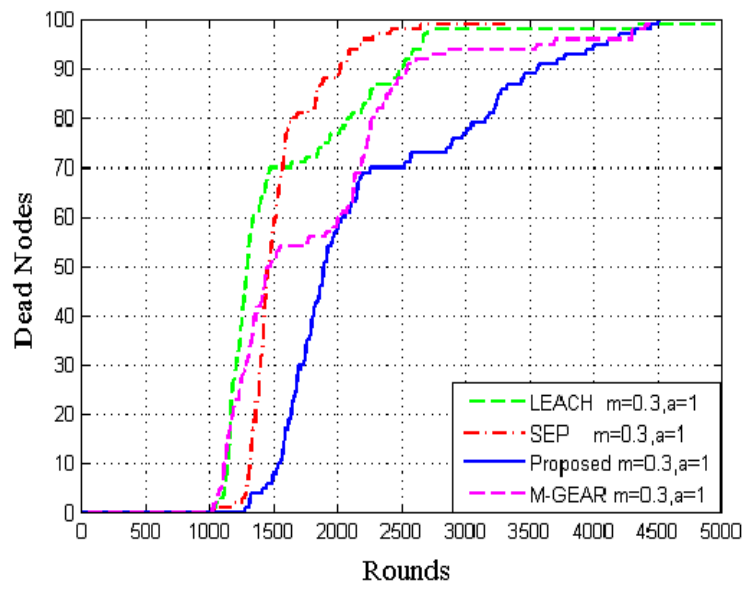

Fig 3: Comparison of proposed algorithm with existing in existence of heterogeneity $(\mathrm{m}=0.3, \mathrm{a}=1)$

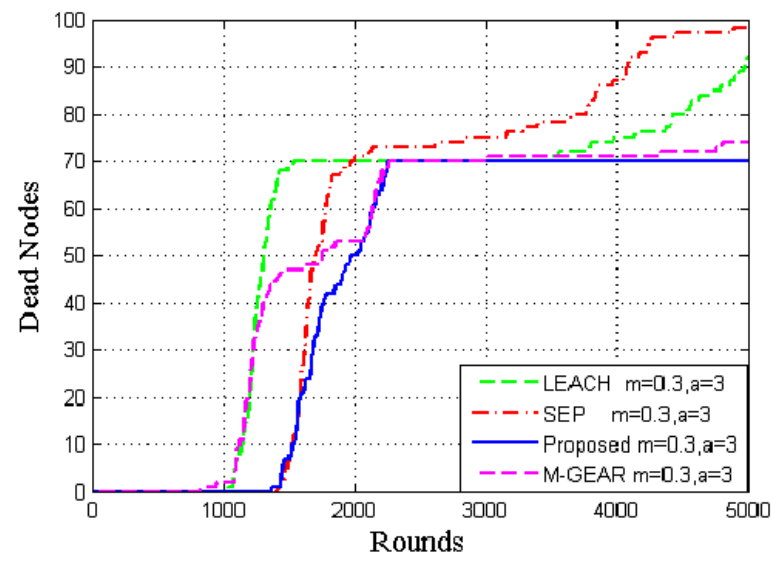

Fig 4: Comparison of proposed algorithm with existing in existence of heterogeneity $(\mathrm{m}=0.3, \mathrm{a}=3)$

\subsection{Throughput Comparison}

This section show packet delivery ratio of overall network as throughput within figure 5 and figure 6 with two different parameter setting as given before.

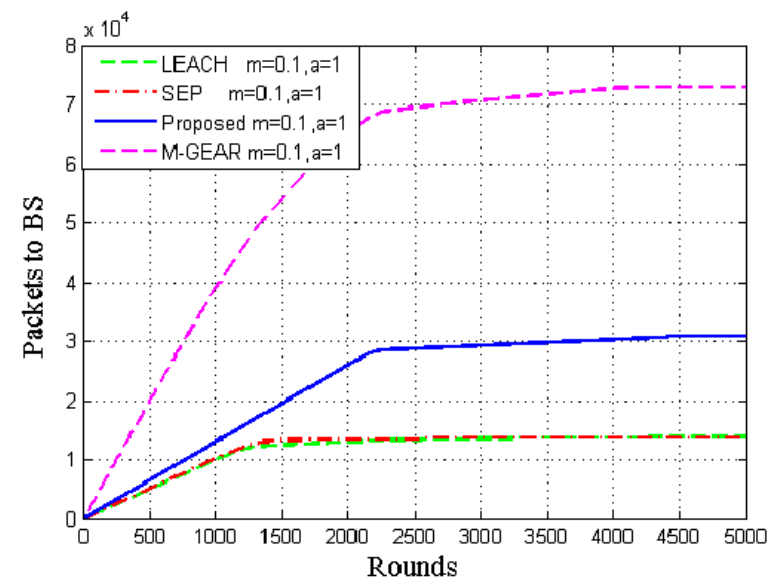

Fig 5. Comparison of proposed algorithm with existing in existence of heterogeneity $(m=0.1, a=1)$

The results for proposed method are better comparing to LEACH and SEP but somehow not enough good as MGEAR. The proposed method guarantees for suitable distribution for cluster head selection as rounds increases. 


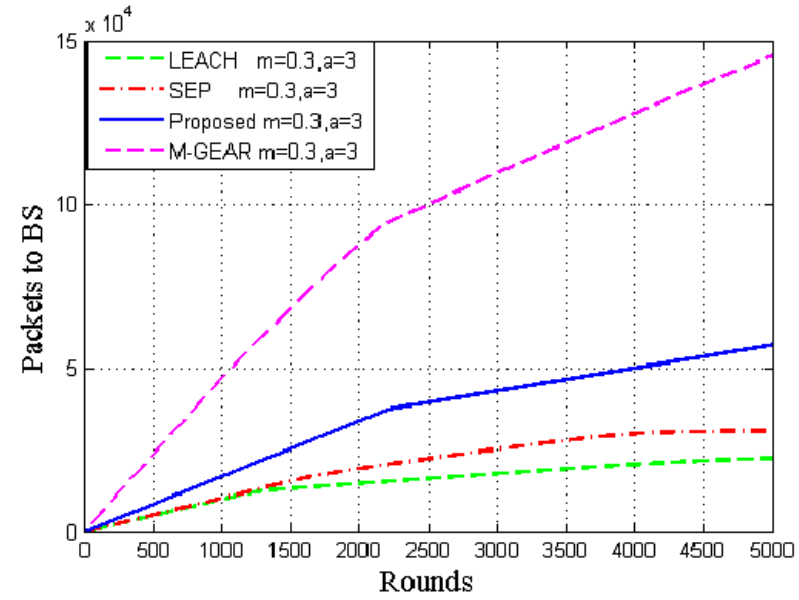

Fig 6. Comparison of proposed algorithm with existing in existence of heterogeneity $(m=0.3, a=3)$

\section{CONCLUSION}

Normally all current algorithms are totally depending on only heterogeneous node distribution because of it increases network complexity. But it's well known that after some time this heterogeneous network automatically becomes homogeneous network as consume all advance more energy node within starting rounds and all nodes are same energy level. Some methods like LEACH and SEP doesn't consider this case but proposed method works very well in both heterogeneous and homogeneous environment. The propose method select $\mathrm{CH}$ on basic of node remaining energy as well as network average energy. Hence WSN network overall average life time will always optimized by better $\mathrm{CH}$ selection. The nodes have more or same energy level as overall network average energy always get selected as $\mathrm{CH}$, so network energy distribution always remain high in compare to other methods. The power switch ON-OFF modes give the energy boost by saving energy of some nodes. This work gives a enhance method for improve network life time, stability of network, remaining energy of node with respective rounds and achieve higher data rates with compare existing energy effective schemes such as SEP, LEACH and MGEAR.

\section{REFERENCES}

[1] R. Mitra and D. Nandy. A survey on clustering techniques for wireless sensor network. Int. J. of Research in Computer Science, 2(4):51-57, 2012.

[2] Y. Khan, N. Javaid, M. J. Khan, Y. Ahmad, M. H. Zubair, S. A. Shah, "LPCH and UDLPCH: Locationaware Routing Techniques in WSNs", IEEE, 2013.

[3] Pei Huang, Chen Wang, and Li Xiao, "Improving Endto-End Routing Performance of Greedy Forwarding in Sensor Networks", IEEE Transactions On Parallel And Distributed Systems, Vol. 23, No. 3, Pages 556-563 IEEE, 2012.

[4] W. Heinzelman, A. Chandrakasan, and H. Balakrishnan, "Energy-efficient routing protocols for wireless microsensor networks," in Proc. 33rdHawaii Int. Conf. SystemSciences(HICSS), Maui, HI,Jan. 2000.

[5] S. Lindsey and C. S. Raghavendra. PEGASIS: power efficient gathering in sensor information systems. In Proceedings of the IEEE Aerospace Conference, March 2002.
[6] X. H. Wu, S. Wang, "Performance comparison of LEACH and LEACH-C protocols by NS2," Proceedings of 9th International Symposium on Distributed Computing and Applications to Business, Engineering and Science. Hong Kong, China, pp. 254-258, 2010

[7] G. Smaragdakis, I. Matta, A. Bestavros, SEP: A Stable Election Protocol for clustered heterogeneous wireless sensor networks, in: Second Interna-tional Workshop on Sensor and Actor Network Protocols and Applications (SANPA 2004), 2004.

[8] L. Qing, Q. Zhu, M. Wang, "Design of a distributed energy-efficient clustering algorithm for heterogeneous wireless sensor networks". ELSE-VIER, Computer Communications 29, 2006, pp 2230- 2237.

[9] S. Lindsey, C.S. Raghavendra, PEGASIS: power efficient gathering in sensor information systems, in: Proceedings of the IEEE Aerospace Conference, Big Sky, Montana, March 2002.

[10] Manjeshwar, D.P. Agrawal, TEEN: a protocol for enhanced efficiency in wireless sensor networks, in: Proceedings of the 1st International Workshop on Parallel and Distributed Computing Issues in Wireless Networks and Mobile Computing, San Francisco, CA, April 2001.

[11] Manjeshwar, D.P. Agarwal, APTEEN: a hybrid protocol for efficient routing and comprehensive information retrieval in wireless sensor networks, in: Parallel and Distributed Processing Symposium. Proceedings International, IPDPS 2002, pp. 195-202.

[12] Ying Liao, Huan Qi, and Weiqun Li, "Load-Balanced Clustering Algorithm with Distributed Self-Organization for Wireless Sensor Networks", IEEE Sensors Journal, Vol. 13, No. 5, May 2013.

[13] M. Chatterjee, S. K. Das, and D. Turgut, "WCA: A weighted clustering algorithms for mobile ad hoc networks,"Cluster Comput., vol. 5, no. 2, pp. 193-204, 2002.

[14] Y. Fernandess and D. Malkhi, "K-clustering in wireless ad-hoc networks," in Proc. 2nd ACM Workshop Principles Mobile Comput. Conf., Oct. 2002, pp. 31-37.

[15] M. Lehsaini, H. Guyennet, and M. Feham, "A novel cluster-based selforganization algorithm for wireless sensor networks," inProc. Int. Symp. Collabor. Technol. Syst. Conf., May 2008, pp. 19-26.

[16] S.K. Mitra and M.K. Naskar. Comparative study of radio models for data gathering in wireless sensor network. N ternational Journal of Computer Applications (09758887), 27(4):49-57, August 2011.

[17] G. Smaragdakis I. Matta and A. Bestavros. SEP: A stable election protocol for clustered heterogeneous wireless sensor networks. in Second International Workshop on Sensor and Actor Network Protocols and Applications (SANPA), 2004.

[18] Q. Nadeem M.B. Rasheed N. Javaid Z.A. Khan Y. Maqsood and A. Din. M-GEAR: Gateway-based energyaware multi-hop ng protocol for wsns. Broadband and Wireless Computing, Communication and Applications (BWCCA) of IEEE, pages $164\{169$, July 2013. 